NATIONAL AERONAUTICS AND SPACE ADMINISTRATION

Technical Memorandum 33-666

\title{
Nonstationary Homogeneous \\ Nucleation
}

K. G. Harstad

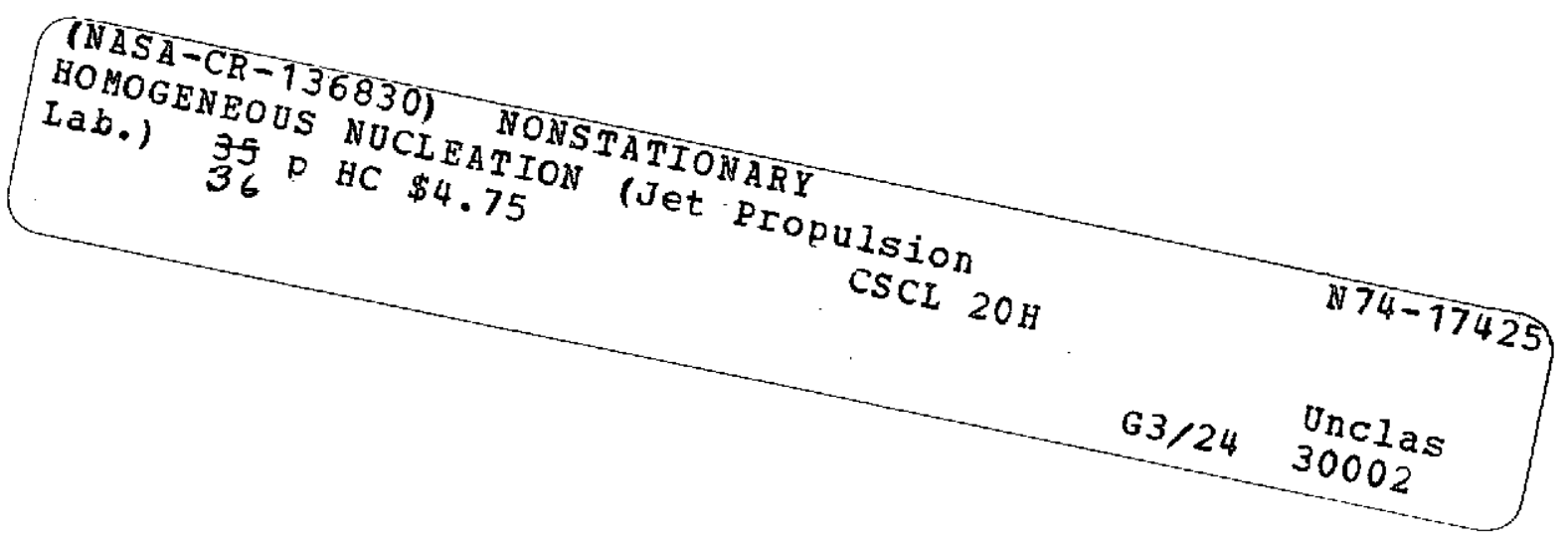

JET PROPULSION LABORATORY CALIFORNIA INSTITUTE OF TECHNOLOGY

PASADENA, CALIFORNIA

January 15,1974 
Technical Memorandum 33-666

\title{
Nonstationary Homogeneous Nucleation
}

\author{
K. G. Harstad
}

JET PROPULSION LABORATORY CALIFORNIA INSTITUTE OF TECHNOLOGY

PASADENA, CALIFORNIA

January 15,1974 
Prepared Under Contract No. NAS 7-100

National Aeronautics and Space Administration 


\section{PREFACE}

The work described in this report was performed by the Propulsion Division of the Jet Propulsion Laboratory. 
CONTENTS

Introduction ............................ . . 1

I. Review of Condensation Theory . . . . . . . . . . . . . . . 1

Thermodynamics and Droplet Equilibrium . . . . . . . . . . . 1

Quasi-equilibrium Cluster Distribution ............ 3

Nucleation Equations .................... . . 6

Equations for Droplet Growth and Energy Balance . . . . . . 10

Nozzle Flows ......................... 12

Comments.......................... 16

II. Solutions to the Transient Equation . . . . . . . . . . . . 17

Conversion of the Nucleation Equation . . . . . . . . . . . . 17

Comparison with the Numerical Results of Courtney and

Clark...................... . . 19

Relaxation in Terms of Universal Parameters . . . . . . . . 22

References . . . . . . . . . . . . . . . . . . . . . . 24

FIGURES

1. Transient behavior of water droplet concentrations for $\mathrm{p} / \mathrm{p}_{\mathrm{s}}=4.5$ and $\mathrm{T}=263^{\circ} \mathrm{K}$................ 26

2. Transient behavior of water droplet concentrations for $\mathrm{p} / \mathrm{p}_{\mathrm{S}}=5.1$ and $\mathrm{T}=263^{\circ} \mathrm{K} \ldots . . . . . . . .$.

3. Transient behavior of water droplet concentrations for $\mathrm{p} / \mathrm{p}_{\mathrm{s}}=20.0$ and $\mathrm{T}=213^{\circ} \mathrm{K}$

4. Transient behavior of water droplet concentrations, same conditions as Fig. 3......................

5. Nondimensional nucleation relaxation time $\theta_{\mathrm{R}} \mathrm{vs}$. critical droplet size $g$ for various values of the surface energy

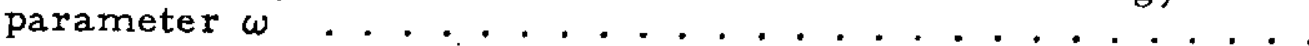

\section{Preceding page blank}




\begin{abstract}
The theory of homogeneous condensation is reviewed and equations describing this process are presented. Numerical computer solutions to transient problems in nucleation (relaxation to steady state) are presented and compared to a prior computation. The present method of computation is much faster than that used previously.
\end{abstract}




\section{Introduction}

This report deals mainly with theory describing the initial stage of condensation of a supersaturated vapor to the liquid phase. There are two types of condensation: heterogeneous and homogeneous. Heterogeneous condensation occurs when the condensation centers, or nuclei, are composed of material other than that of the vapor of interest. Examples of such nuclei are ions, dust particles, and droplets of an easily condensible impurity vapor that may be present. This type of condensation is in general quite complex and little is known about it (Ref. 1). If the vapor is sufficiently pure and free of foreign particles, then a spontaneous condensation, or nucleation, may occur. The growth of these self-nuclei leads to homogeneous condensation. This process tends to become important if the supersaturation condition arises rapid1y. It is the process of homogeneous nucleation that is amenable (at least to a certain extent) to mathematical modeling and is discussed here. The model equations are well known and are reviewed in the first part of the report. In a second part, some numerical solutions to problems dealing with a nonsteady state are discussed. I. Review of Condensation Theory Thermodynamics and Droplet Equilibrium

Consider a system at given pressure $\mathrm{p}$ and temperature $\mathrm{T}$. From thermodynamics (Refs, 1-3), the system is in equilibrium when the Gibbs free energy $G\left(\mathrm{p}, \mathrm{T}, \mathrm{c}_{\mathrm{i}}\right)=\mathrm{U}+\mathrm{pV}-\mathrm{TS}$ is a minimum. Mole fraction of phase $i$ is $c_{i}$, molar quantities are internal energy $U$, volume $V$ and entropy $s$. The chemical potential of phase $i$ is $\mu_{i}=\frac{\partial G}{\partial c_{i}}$ with $G=\sum_{i} c_{i} \mu_{i}$. 
The chemical potential can be regarded as the partial Gibbs energy of the phase. The equilibrium condition is

$$
\mathrm{dG}=\mathrm{Vdp}-\mathrm{Sd} \mathrm{T}+\sum_{\mathbf{i}} \mu_{\mathrm{i}} \mathrm{dc} \mathrm{c}_{\mathbf{i}}=0
$$

For a two phase system, liquid (l) and vapor (v),

$$
\begin{aligned}
& d c_{v}+d c_{\ell}=0, \\
& v d p-s d T+\mu_{v} d c_{v}+\mu_{\ell} d c_{\ell}=0 .
\end{aligned}
$$

For constant $\mathrm{p}$ and $\mathrm{T}$, equilibrium requires $\mu_{\mathrm{V}}=\mu_{\ell}$. Consider an isothermal reversible process. $V=\sum_{i} c_{i} V_{i}$ and $d_{\mu_{i}}=V_{i}$ dp from the second form of $\mathrm{G}$.

$$
\mathrm{d}\left(\mu_{\ell}-\mu_{\mathrm{v}}\right)=\left(\mathrm{v}_{\ell}-\mathrm{v}_{\mathrm{v}}\right) \mathrm{dp} \simeq-\mathrm{v}_{\mathrm{v}} \mathrm{d} \mathrm{p}
$$

since $\mathrm{V}_{\mathrm{v}} \gg \mathrm{V}_{\ell}$. For a perfect gas, $\mathrm{V}_{\mathrm{v}}=\mathrm{RT} / \mathrm{p}$ and

$$
\mu_{\ell}-\mu_{v}=R T \ell n\left(p_{s} / p\right)
$$

where $p_{S}(T)$ is the saturation vapor pressure for a flat surface. This relation for the deviation of the chemical potentials from the equilin brium condition is used to develop an expression for the energy of droplet formation. For a system of $\mathrm{N}$ molecules of which $\mathrm{g}$ form a liquid drop, the free energy is

$$
\phi_{\mathrm{g}}=(\mathrm{N}-\mathrm{g}) \phi_{\mathrm{v}}+\mathrm{g} \phi_{l}+4 \pi \mathrm{r}^{2} \sigma
$$

where $\phi_{i}\left(\sim_{i}\right)$ is the free energy per particle, r the drop radius, and $\sigma(\mathrm{T})$ the surface tension (energy). The free energy of formation

$$
\Delta \phi_{\mathrm{g}}=\phi_{\mathrm{g}}-\phi_{\mathrm{o}}=\mathrm{g}\left(\phi_{\mathrm{l}}-\phi_{\mathrm{v}}\right)+4 \pi \mathrm{r}^{2} \sigma \text {. }
$$

If $m$ is the molecular mass and $\rho_{\ell}$ the liquid density, $m g=\frac{4}{3} \pi r^{3} \rho_{\ell}$; also, $\phi_{l}-\phi_{\mathrm{v}}=\mathrm{kT} \ln \left(\mathrm{p}_{\mathrm{s}} / \mathrm{p}\right)$. Substituting, 


$$
\Delta \phi_{g}(\mathrm{p}, \mathrm{T}, \mathrm{g})=\operatorname{gkT\ell n}\left(\mathrm{p}_{\mathrm{s}} / \mathrm{p}\right)+(4 \pi)^{1 / 3}\left(3 \mathrm{mg} / \rho_{\ell}\right)^{2 / 3} \sigma
$$

Droplet equilibrium requires $\frac{\partial}{\partial g}\left(\Delta \phi_{g}\right)=0$, or

$$
\mathrm{kT} \ln \left(\mathrm{p} / \mathrm{p}_{\mathrm{s}}\right)=\frac{2 \mathrm{mg}}{\rho_{\ell} \mathrm{r}} .
$$

This formula defines an unstable condition since the droplet free energy is maximum, not minimum. For a drop of given radius $r$, the above rela tion gives the saturation pressure $p$ of the drop. (As $\left.r \rightarrow \infty, p \rightarrow p_{s} \cdot\right)$ For given pressure $\mathrm{p}>\mathrm{P}_{\mathrm{S}}$, the vapor is undersaturated with respect to a drop of radius smaller than $r$ (hence such a drop will evaporate) and supersaturated with respect to a drop of radius larger than $r$ (such a drop will tend to grow). If $\mathrm{p} \leq \mathrm{p}_{\mathrm{s}}$, all drops will tend to evaporate, that is, the vapor is undersaturated with respect to drops of all sizes. In a supersaturated vapor $\left(p>p_{s}\right)$, drops of size $r$ are the critical sized nuclei that initiate the process of homogeneous condensation. The superscript "*" will be used to denote this critical size. Quasi-equilibrium Cluster Distribution

Assuming a finite probability for a vapor molecule sticking to another molecule or molecular cluster upon collision, thermal fluctuations in a vapor will lead to droplet formation. classical thermodynamics or statistical mechanics gives the following result for the quasi-equilibrium ${ }^{*}$ distribution of clusters or droplets (Refs. 1, 2, 4-12) :

$$
\mathrm{N}_{\mathrm{g}}=\mathrm{C} \exp \left(-\Delta \phi_{\mathrm{g}} / \mathrm{kT}\right) \text {. }
$$

This distribution, along with a binary collision assumption, leads to the classical liquid drop theory of nucleation. The Boltzmann-1ike

\footnotetext{
"Strict thermodynamic equilibrium occurs only for $p=p_{s}$.
} 
distribution represents a balance between the effect of thermal collisions and the tendency for droplets to vaporize - it holds when the vapor is undersaturated with respect to the drops. It cannot be considered valid if $\mathrm{p}>\mathrm{p}_{\mathrm{s}}$ and $\mathrm{r}>\mathrm{r}^{*}$ (see Ref. 2). Initially held was the view that the proportionality factor $C$ is nearly equal to the vapor number density (Refs. 1,2). In Refs. 4 and 13 it is suggested that taking proper account of the cluster partial pressure results in a modification of the factor to the saturation number density (this is equivalent to replacing $g$ by $(g-1)$ in the bulk energy change term in $\left.\Delta \phi_{g}\right)$. Controversy over proper treatment of rotational and translational partition functions of a cluster (Refs. 1, 5-12) has been sustained over the past ten years. Extremely large differences in $\mathrm{C}$ estimates (as large as 17 orders of magnitude, Ref. 5) resulted. The latest references (Refs. 9-12) seem to have resolved the problem; the factor $C$ is ascertained to be roughly equal to the liquid number density. It should be noted that the classical thermodynamic representation of clusters as droplets can only be valid for $g \gg 1$. Unfortunately, for typical conditions, $\mathrm{g}^{*} \leqslant 100$. On the microscale relevant to the nucleation problem, a detailed quantum mechanical description of the interaction between molecules of a cluster is needed in order to correctly calculate the energy of formation, heat capacity, etc. This is an impossibly difficult approach. The extension of the classical liquid drop theory to small clusters can be justified, however, on the basis of the agreement of the resulting steady state nucleation rates with experiment (Refs, 14-17). This agreement requires that the original liquid 
drop theory $(\mathrm{C} \approx$ vapor number density) be modified by choosing a (sma1ler) surface tension 'to account for the effect of surface curvature' (Refs. $14,15,18$ ) or by taking $\mathrm{C} \approx$ liquid number density (Ref. 16). Either modification will give roughly the same result; the 1atter seems to have a better theoretical justification (above paragraph). Further discussion on the extension of drop theory can be found in Ref. 1. Recently, there have been efforts to study the behavior of small clusters by computer techniques. Monte Carlo calculations using a lattice gas (Ref. 19) for $g \leq 1000$ have shown that the surface energy (area) varies as the macroscopic dependence $\mathrm{g}^{2 / 3}$ unless the droplet size is much smaller than critical or the nucleation rate is very high. Several references deal with atoms interacting classically through the Lennard-Jones 12-6 potential. Computer calculation of cluster configurational integrals that are related to the internal cluster partition functions yields good agreement between the resulting (discrete) saturation pressures for $2 \leq \mathrm{g} \leq 14$ and the thermodynamic expression for droplet equilibrium (Ref. 20). This fortuitous result furnishes further credence for the use of liquid drop theory; however, there is some doubt as to the agreement that may be expected for larger $g$ (Ref. 20). In Ref. 21 the Monte Carlo technique is applied to cluster integrals to compute the Helmholtz free energy for $13 \leq \mathrm{g} \leq 100$ and $0 \leq \mathrm{T} \leq 100^{\circ} \mathrm{K}$ using argon potential constants. Essentially the same problem (with $\left.15 \leq \mathrm{g} \leq 100, \mathrm{~T} \leq 75^{\circ} \mathrm{K}\right)$ is approached in Ref. 22 by simulation of the cluster molecular dynamics; presented are graphs of the Gibbs free energy and other thermodynamic quantities. Similar efforts in the near future may lead to a definitive validation of liquid drop theory or to a more 
realistic model for the free energy and cluster distribution of smaller clusters; for the present, the liquid drop model is deemed adequate. Nucleation Equations

The droplet formation or nucleation equations are formed assuming that only binary collisions between a droplet and a vapor molecule (monomer) are important, i.e., the droplet size changes by addition or subtraction of a single molecule at a time. This means that the vapor must be sufficiently tenuous and that the concentration of dimers and higher order polymer clusters must be much smaller than the monomer concentration. The sticking ability of a molecule upon collision with a droplet is characterized by a condensation accomodation coefficient $\alpha_{c}$. The free molecular flow particle flux is $\beta=\mathrm{p} / \sqrt{2 \pi \mathrm{mkT}}$ and the drop area is $4 \pi r^{2}=g^{2 / 3} A_{c}$ where $A_{c}=(4 \pi)^{1 / 3}\left(3 m / \rho_{\ell}\right)^{2 / 3}$ is a characteristic molecular area. The droplet growth collision frequency is thus $\nu_{g}=g^{2 / 3}$. $\nu_{c} ; \nu_{c} \equiv \alpha_{c} \beta A_{c}$. The evaporation rate $\nu_{g}^{(e)}$ is given by the principle of detailed balancing using the quasi-equilibrium cluster distribution:

$$
\nu_{g}^{(e)}=\left(N_{g-1} / N_{g}\right)_{\text {quasi-equi1. }} \nu_{g-1}=\exp \left(\frac{\Delta \phi_{g}-\Delta \phi_{g-1}}{k T}\right) \nu_{g-1} .
$$

The rate of change of the concentration of droplet size $g$ is given by

$$
\frac{d N}{d t}=\nu_{g-1} N_{g-1}+v_{g+1}^{(e)} N_{g+1}-\left(\nu_{g}+\nu_{g}(e)\right) N_{g} \text {. }
$$

A nondimensional time variable is defined by $d \theta \equiv \nu_{c}(t) d t$. (Variables $\mathrm{p}$ and $\mathrm{T}$ are known functions of time.)

Then

$$
\frac{\mathrm{dN}}{\mathrm{d \theta}}=\zeta_{g-1} f_{g-1}+\zeta_{g} f_{g+1}-\left(\zeta_{g}+\zeta_{g-1}\right) f_{g}
$$


where $\quad \mathbf{f}_{\mathrm{g}} \equiv \mathrm{N}_{\mathrm{g}} \exp \left(\Delta \phi_{\mathrm{g}} / \mathrm{kT}\right)$ and $\zeta_{\mathrm{g}} \equiv \mathrm{g}^{2 / 3} \exp \left(-\Delta \phi_{\mathrm{g}} / \mathrm{kT}\right)$.

This can be written as

$$
\frac{\mathrm{dN}}{\mathrm{d} \theta}=I_{g-1}-I_{g}, \quad I_{g} \equiv \zeta_{g}\left(f_{g}-f_{g+1}\right) .
$$

$I_{g}$ is the net flux of droplets making the transition $g \rightarrow(g+1)$. The nucleation rate is determined by $I_{g} *$. Let $y_{g}$ denote the total number of droplets of size greater than $g$,

then $I_{g}=\frac{d y}{d \theta}$. The nucleation rate is thus found by solving for the

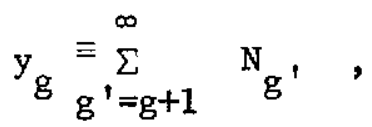

variable set $\mathrm{N}_{g}$ or $\mathrm{y}_{\mathrm{g}}$ and using the equation of droplet equilibrium to calculate $\mathrm{g}^{*}$.

Since $g \gg 1$ (at least for cluster sizes which correspond to physically realistic situations in gases), the discrete variable $g$ may be considered continuous to a good approximation (see Ref. 23). Correspondingly, the differences indicated in the above equations can be considered as differentials :

$$
\frac{\partial N_{g}}{\partial \theta}=-\frac{\partial I}{\partial g} \text { and } I=-5 \frac{\partial f}{\partial g} \text {, }
$$

or

$$
\frac{\partial N_{g}}{\partial \theta}=\frac{\partial}{\partial g}\left[g^{2 / 3}\left(\frac{\partial N_{g}}{\partial g}+\frac{N_{g}}{k T} \frac{\partial \Delta \phi}{\partial g}\right)\right] .
$$

It is convenient to define a new 'area' variable $\xi \equiv \frac{3}{2} \mathrm{~g}^{2 / 3}$. Also let $\mathrm{N}_{\xi} \equiv \mathrm{g}^{1 / 3} \mathrm{~N}_{\mathrm{g}}$ and $\mathrm{F} \equiv \frac{\Delta \phi_{\mathrm{g}}}{\mathrm{kT}}-\frac{1}{3} \ln \mathrm{g}$. Then

$$
\mathrm{N}_{\xi, \text { quasi-equil. }}=\mathrm{C} \exp (-\mathrm{F}) \text {, }
$$




$$
\begin{aligned}
& y=\int_{g}^{\infty} \mathrm{N}_{g} d g=\int_{\xi}^{\infty} \mathrm{N}_{\xi} \mathrm{d} \xi, I=\frac{\partial y}{\partial \theta}, \\
& \frac{\partial \mathrm{N}_{E}}{\partial \theta}=\frac{\partial}{\partial \xi}\left(\frac{\partial \mathrm{N}_{\xi}}{\partial \xi}+\mathrm{N}_{\xi} \frac{\partial \mathrm{F}}{\partial \xi}\right)=-\frac{\partial \mathrm{I}}{\partial \xi},
\end{aligned}
$$

and

$$
\frac{\partial y}{\partial \theta}-F, \xi \frac{\partial y}{\partial \xi}=\frac{\partial^{2} y}{\partial \xi^{2}}
$$

The last equation is a one dimensional convection-diffusion equation with convection velocity - $\frac{\partial F}{\partial \xi}$. The presentation of the liquid drop nucleation theory in terms of difference and/or differential equations is now complete. The steady state solution or long time behavior of the differential equations is given next. The development will parallel that given in Ref. 2 .

In the limit $\frac{\partial N_{\xi}}{\partial \theta} \rightarrow 0, I=I(\theta)$,

$$
\frac{\partial N_{\xi}}{\partial \xi}+N_{\xi} \frac{\partial F}{\partial \xi}=-I(\theta)
$$

and

$$
\mathrm{N}_{\xi}=I \exp (-\mathrm{F}) \int_{\xi}^{\infty} \exp (\mathrm{F}) \mathrm{d} \xi \text {. }
$$

In a supersaturated vapor, $F$ has a maximum at $\xi=\xi^{*}$. Let $\mu \equiv \ln \left(p_{s} / p\right)$ and $\omega(\mathrm{T}) \equiv \sigma \mathrm{A}_{\mathrm{c}} / \mathrm{kT}$, then

$$
F=\left(\frac{2}{3} \xi\right)^{3 / 2} \mu+\left(\frac{2}{3} \xi\right) \omega-\frac{1}{2} \ln \left(\frac{2}{3} \xi\right)
$$

Let $\Delta_{0} \equiv \frac{9 \mu^{2}}{8 \omega}=\Delta(1-\Delta)^{2}, \xi^{*}=\frac{2}{3}\left(\frac{\omega}{\mu}\right)^{2}(1-\Delta)^{2}, x=\frac{\mu^{2}}{4 \omega} \frac{(1-3 \Delta)}{(1-\Delta)^{2}}$.

Expanding $\mathrm{F}$ in a Taylor series about $\xi^{*}$,

$$
\mathrm{F}=\mathrm{F}^{*}-x\left(\xi-\xi^{*}\right)^{2}+\text { higher order terms. }
$$


Using the method of steepest descent, this expression is substituted in the above integral giving

$$
N_{\xi} \simeq \frac{1}{2} I \sqrt{\pi / x} \exp \left(F^{*}-F\right) \operatorname{erfc}\left(\sqrt{\mu}\left(\xi-\xi^{*}\right)\right)
$$

As $\xi \rightarrow 0$, then $\mathrm{N}_{\xi} \rightarrow \mathrm{N}_{\xi}$, quasi-equil. and erfc $\simeq 2$,

$$
\therefore C=I \sqrt{\pi / x} \exp \left(F^{*}\right)
$$

Finally, for steady state,

$$
I=\frac{1}{3} C \sqrt{\omega(1-3 \Delta) / \pi} \exp \left(-\frac{\Delta \phi_{g}^{*}}{k T}\right)
$$

and $\quad \mathrm{N}_{\mathrm{g}}=\frac{1}{2}\left(\mathrm{~N}_{\mathrm{g}, \text { quasi-equil. }}\right)$ erfc $\left(\sqrt{u}\left(\xi-\xi^{*}\right)\right)$.

Typically, $\Delta \approx \Delta_{0} \ll 1$ and the steady state value of $I$ is approximately $\frac{2}{3} \sqrt{\omega / \pi}$ times the critical droplet concentration (times the characteristic frequency $\nu_{c}$ if reference is made to dimensional t. rather than $\theta$ ). Also note that the critical concentration in steady state is half the quasi-equilibrium value. For $\xi \gg \xi^{*}$, the expression for $\mathrm{N}_{g}$ is only qualitatively correct.

A gas that is immiscible with or has no affinity for the liquid phase in consideration will be called an inert gas. It is not expected that the inclusion of an inert gas in the system will have any effect on nucleation (Refs. 4, 18); this is confirmed by experiment (Refs. 14, 16, 17). Of course, variable p will then be identified with the partial pressure of the vapor phase. 
Equations for Droplet Growth and Energy Balance

Once a critical sized nucleus has formed, it will grow to form a condensation drop. Initially, this is a one-way process and tends to be fast. As each molecule adds its mass to the drop, it also brings its latent heat of evaporation, a quantity much larger than $\mathrm{kT}$. The drop quickly increases in temperature with consequent increase in its vapor pressure and evaporation again becomes important. An inert gas present can act as a heat sink for the latent heat (through thermal relaxation by collisions with drops) and thus can increase the condensation rate after nucleation (Ref. 18). Equations describing these processes in the free molecular flow limit are given in this section (see also Ref. 1). It is assumed that there is no slip of the drop in the flow.

Subscript "d" denotes drop quantities, subscript "p" the inert parent or carrier gas and subscript "e" an exiting or evaporating molecule. The heat flux to the drop is

$$
\begin{aligned}
q_{d} & =\alpha_{t c}\left[n_{v} k T\left(\frac{2 k T}{\pi m}\right)^{\frac{1}{2}}-n_{v e} k T d\left(\frac{2 k T d}{\pi m}\right)^{\frac{1}{2}}\right] \\
+ & \alpha_{t p}\left[n_{p} k T\left(\frac{2 k T}{\pi m p}\right)^{\frac{1}{2}}-n_{p e} k T d\left(\frac{2 k T d}{\pi m p}\right)^{\frac{1}{2}}\right],
\end{aligned}
$$

where $\alpha_{t c}$ and $\alpha_{t_{p}}$ are thermal accommodation coefficients and $n$ denotes number density. Since the parent gas is inert, $n_{p e}=n_{p} \sqrt{T / T} d$ From droplet equilibrium

$$
n_{v e}{ }^{k T} T_{d}=p_{s d}=p_{s}\left(T_{d}\right) \exp \left(\frac{2 m \sigma\left(T_{d}\right)}{p_{f} k T_{d} r}\right) .
$$


The drop energy equation is (for constant $\rho_{\ell}, r$ continuous)

$$
q_{d}=\rho_{\ell}\left(\Delta H \frac{d r}{d t}+\frac{1}{3} r_{\ell} \frac{d T}{d t}\right)
$$

where $\Delta \mathrm{H}$ is the energy change per unit added mass and $\mathrm{C}_{\ell}$ is the liquid specific heat at constant pressure. The drop growth rate is

$$
\rho_{\ell} \frac{\mathrm{d} r}{\mathrm{dt}}=\frac{1}{2} \alpha_{c} \mathrm{~m}\left[\mathrm{n}_{\mathrm{v}}\left(\frac{2 \mathrm{kT}}{\pi \mathrm{m}}\right)^{\frac{1}{2}}-\mathrm{n}_{\mathrm{ve}}\left(\frac{2 \mathrm{kT} d}{\pi \mathrm{m}}\right)^{\frac{1}{2}}\right] \text {. }
$$

To calculate $\Delta H$, consider the three step process of (1) compressing the vapor to the drop saturation pressure (T constant), (2) change of phase with latent heat $\mathrm{L}$ and (3) heating from $\mathrm{T}$ to $\mathrm{T}_{\mathrm{d}}$.

$$
\Delta \mathrm{H}=\left[\frac{k \mathrm{~T}}{\mathrm{~m}} \ln \left(\frac{\mathrm{p}_{\mathrm{s}}(\mathrm{T})}{\mathrm{p}_{\mathrm{v}}}\right)+\frac{2 \sigma(\mathrm{T})}{\rho_{\ell} \mathrm{T}}\right]-\mathrm{L}(\mathrm{T})+\mathrm{c}_{\ell}\left(\mathrm{T}_{\mathrm{d}}-\mathrm{T}\right) .
$$

If the heating is done first on the vapor phase before the compression and phase change, then

$$
\Delta H=C_{v}\left(T_{d}-T\right)+\left[\frac{k T_{d}}{m} \ln \left(\frac{p_{s}\left(T_{d}\right)}{p_{v}}\right)+\frac{2 \sigma\left(T_{d}\right)}{\rho_{\ell} r}\right]-L\left(T_{d}\right),
$$

where the vapor constant pressure specific heat $C_{v}=\frac{5 k}{2 m}$ for a monatomic vapor. It is to be noted that for a perfect gas and neglecting the liquid specific volume compared to that of the vapor (Ref. 3),

$$
\frac{\mathrm{dL}}{\mathrm{dT}} \approx \mathrm{C}_{\mathrm{v}}-\mathrm{C}_{\ell}
$$

and

$$
\mathrm{L} \simeq \frac{\mathrm{kT}^{2}}{\mathrm{~m}} \frac{\mathrm{d} \ell \mathrm{nP}_{\mathrm{s}}}{\mathrm{dT}}
$$

The first equation implies $\mathrm{C}_{\ell}\left(\mathrm{T}_{\mathrm{d}}-\mathrm{T}\right)-\mathrm{L}(\mathrm{T})=\mathrm{C}_{\mathrm{v}}\left(\mathrm{T}_{\mathrm{d}}-\mathrm{T}\right)-\mathrm{L}\left(\mathrm{T}_{\mathrm{d}}\right)$. The second is known as clapeyron's relation for a vapor. The brackets 
in each expression for $\Delta H$ enclose terms giving the work of compression. This compression work is nu11 for a drop of critical size. Further, each individual term in a bracket decreases in magnitude with increasing time (increasing drop size and decreasing supersaturation) and tend to be small compared to the latent heat (mL $\gg \mathrm{kT}_{\mathrm{d}}$ ). An approximate expression for the energy change is

$$
\Delta \mathrm{H} \simeq \mathrm{C}_{\ell}\left(\mathrm{T}_{\mathrm{d}}-\mathrm{T}\right)-\mathrm{L}(\mathrm{T})
$$

The differential equations for drop size $r$ and temperature $\mathrm{T}_{\mathrm{d}}$ depend on the parameters $\mathrm{T}, \mathrm{n}_{\mathrm{v}}$, and $\mathrm{n}_{\mathrm{p}}$.

Formulas giving the effect of droplet slip relative to the gas are found in Ref. 1; consideration of this in nozzles or for other dynamic systems is not likely to be necessary. Discussions and calculations relating to a finite knudsen number are given in Refs. 1 and 24; again, this is not likely to be important in nozzles (Refs. 15, 16). Accommodation coefficients are listed in Refs. 1 and 25. A parametric study on the effects of heat transfer on drop growth is conducted in Ref. 26. Both the nucleation rate and the growth rate are important to the condensation problem. (Refs. 18, 26).

Nozzle Flows

This review of condensation theory topics will conclude by giving attention to a particular situation where condensation may be important that of flow in a nozzle. The flow is taken to be one dimensional and steady. The axial coordinate of the nozzle $x$, velocity is $u(x)$ and $\frac{d}{d t}=u(x) \frac{d}{d x}$. Since a relatively small amount of the gas flow changes phase during condensation, the effects of changes of composition (on 
specific heats, effective gas constant, etc.) will be neglected except for the volume heat addition that results from release of the latent heat. Let $Q(x)$ be the total heat addition, $A(x)$ the prescribed nozzle area and $\rho$ the gas mixture density. Then

$$
\begin{aligned}
& p^{u A}=w=\text { constant, } \\
& \text { pudu }+d_{p}=0, \\
& d Q=C_{p} d T+\text { udu, } \\
& p=\rho^{R T} .
\end{aligned}
$$

Here $C_{P}$ is the mixture constant pressure specific heat and $R$ the mixture effective gas constant. Assuming perfect gas components with specific heat ratios $\gamma_{i}$ and mole fractions $c_{i}$,

$$
\mathrm{R}=\mathrm{k} / \mathrm{i}_{\mathrm{i}} \mathrm{m}_{\mathrm{i}} \mathrm{c}_{\mathrm{i}}
$$

and

$$
\mathrm{C}_{\mathrm{p}}=\mathrm{R}_{i} \mathrm{c}_{i} \gamma_{i} /\left(\gamma_{i}-1\right)
$$

The specific heat ratio for the mixture is $y=C_{p} /\left(C_{p}-R\right)$. The partial vapor pressure is proportional to the total pressure, $\mathrm{p}_{\mathrm{v}}=\mathrm{c}_{\mathrm{v}} \mathrm{p}$. The speed of sound $a=\sqrt{\gamma R T}$ and the Mach number $M=u / a$. Entropy $S(x)=$ $\int \mathrm{d}(\mathrm{x}) / \mathrm{T}(\mathrm{x})$. Solving in terms of $\mathrm{p}$ and $\mathrm{T}$ :

$$
\begin{aligned}
& \rho=\mathrm{p} / \mathrm{RT}, \quad \mathrm{u}=\mathrm{wRT} / \mathrm{pA}, \\
& \mathrm{S}=\mathrm{C}_{\mathrm{p}} \ln \left(\mathrm{T} / \mathrm{T}_{\mathrm{o}}\right)-\operatorname{Rln}\left(\mathrm{p} / \mathrm{p}_{\mathrm{o}}\right), \\
& \mathrm{Q}=\mathrm{C}_{\mathrm{P}}\left(\mathrm{T}-\mathrm{T}_{\mathrm{o}}\right)+\frac{1}{2}(\mathrm{wR} / \mathrm{A}) \mathrm{T}^{2} / \mathrm{p}^{2} .
\end{aligned}
$$

Subscript "o" refers to stagnation or reservoir conditions $(A \rightarrow \infty)$. Let $\alpha_{1} \equiv \exp (\mathrm{S} / \mathrm{R})$ and $\alpha_{2} \equiv 1+\mathrm{Q} / \mathrm{C}_{\mathrm{p}} \mathrm{T}_{\mathrm{o}}$. For simplicity, it is assumed that condensation occurs downstream of the nozzle throat and that the subsonic flow is isentropic $\left(\alpha_{1}=\alpha_{2}=1\right)$. Using subscript " $t$ " to denote throat (sonic) conditions, 


$$
\begin{aligned}
& w=A_{t}\left(\frac{2}{\gamma+1}\right)^{\frac{\gamma+1}{2(\gamma-1)}} p_{o} \sqrt{\gamma / R T_{0}} \\
& T_{t}=T_{o}\left(\frac{2}{\gamma+1}\right) \text { and } p_{t}=p_{0}\left(\frac{2}{\gamma+1}\right)^{\frac{\gamma}{\gamma-1}} .
\end{aligned}
$$

Downstream,

$$
\begin{aligned}
& \mathrm{T} / \mathrm{T}_{\mathrm{o}}=\alpha_{2} /\left(1+\frac{\gamma-1}{2} \mathrm{M}^{2}\right), \\
& \mathrm{p} / \mathrm{p}_{\mathrm{o}}=\left[\alpha_{2} /\left(1+\frac{\gamma-1}{2} \mathrm{M}^{2}\right)\right]^{\frac{\gamma}{\gamma-1}} / \alpha_{1},
\end{aligned}
$$

where $M$ is given by

$$
A / A_{t}=\frac{\alpha_{1}}{M}\left[\left(\frac{2}{\gamma+1}+\frac{\gamma-1}{\gamma+1} M^{2}\right) / \alpha_{2}\right]^{\frac{\gamma+1}{2(\gamma-1)}} .
$$

Given $A, \alpha_{1}$ and $\alpha_{2}$, all other quantities are readily found. The flow is not choked by the heat addition if

$$
\alpha_{2}\left(\frac{A}{\alpha_{1}^{A}{ }^{A}}\right)^{\frac{2(\gamma-1)}{\gamma+1}}>1
$$

(Heat addition during the subsonic portion of the flow changes the location of the sonic point and alters the Mach number-area relationship by $A_{t} \rightarrow A_{s}, \alpha_{1} \rightarrow \alpha_{1} / \alpha_{1 s}$ and $\alpha_{2} \rightarrow \alpha_{2} / \alpha_{2 s}$ where "s" denotes the sonic point.)

Neglecting the sma11 energy change due to the nucleation process, the equation for heat addition is

$$
\begin{aligned}
\rho \mathrm{u} \frac{\mathrm{dQ}}{\mathrm{dx}}=- & 4 \pi \sum_{\mathrm{g}} \mathrm{N}_{\mathrm{g}} \mathrm{q}_{\mathrm{d}}(\mathrm{g}) \mathrm{r}^{2}(\mathrm{~g}) . \\
& (\text { a11 condensation drops) }
\end{aligned}
$$

Once $\mathrm{N}_{\mathrm{g}}$ is specified, the formulation of the nozzle flow problem is complete. The growth rate equation may be written in the dimensionless form 


$$
\frac{\mathrm{d} r}{\mathrm{dx}}=\Gamma(\mathrm{x}, \mathrm{r})=\frac{\alpha_{\mathrm{c}}}{\rho_{\ell}^{\mathrm{u}}}\left[\mathrm{n} \mathrm{v}\left(\frac{\mathrm{mkT}}{2 \pi}\right)^{\frac{1}{2}}-\mathrm{n}_{\mathrm{ve}}\left(\frac{\mathrm{mkT} \mathrm{d}}{2 \pi}\right)^{\frac{1}{2}}\right] .
$$

Let $x_{s}$ denote the saturation $\left(p=p_{s}\right)$ point or starting point (whichever is furthest downstream) and $x_{n}$ a particular nucleation point,

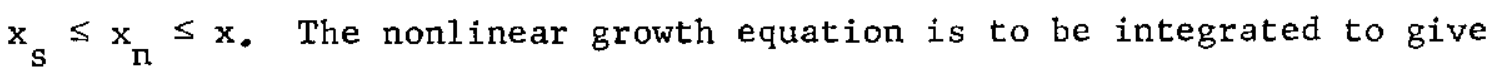
$r=\bar{r}\left(x_{1} x_{n}\right)$ where $\bar{r}\left(x_{n}, x_{n}\right) \equiv r^{*}\left(x_{n}\right)$. Only those values of $x_{n}$ such that $\bar{r}\left(x, x_{n}\right) \geq r^{*}(x)$ can give growing drops. For given $x$, an interval $\Delta x_{n}$ is defined at $x_{n}$ such that $\bar{g}\left(x, x_{n}\right)$ has a value of $g_{n} \pm \frac{1}{2}$ at either endpoint of the interval where $g_{n}$ is an integer and $\left|\vec{g}-g_{n}\right| \leq \frac{1}{2}$ in $\Delta x_{n}$. If $N^{*}$ is the number of condensation nuclei formed at $x_{n}$ in $\Delta x_{n}$,

$$
N_{g}(x)=\sum_{x_{n}} N^{*}\left(x_{n}\right) \delta\left[g-g_{n}\left(x, x_{n}\right)\right]
$$

where $\delta$ is the Dirac delta function. Also,

$$
\frac{d Q}{d x}=-\frac{A_{c} A(x)}{w} \sum_{x_{n}} N^{*}\left(x_{n}\right) g_{n}{ }^{2 / 3} q_{d}\left(g_{n}\right)
$$

The rate of nuclei formation is

$$
\frac{d}{d t} y\left(g^{*}, t\right)=\nu_{c} I^{*}-N_{g} * \frac{d g^{*}}{d t}
$$

therefore

$$
\mathrm{N}^{*}\left(\mathrm{x}_{\mathrm{n}}\right)=\int_{\Delta \mathrm{x}_{\mathrm{n}}}\left(\frac{\nu_{\mathrm{c}}}{\mathrm{u}} \mathrm{I}^{*}-\mathrm{N}_{\mathrm{g}} * \frac{\mathrm{dg}}{\mathrm{dx}}\right) \mathrm{dx}
$$

The integer function $g_{n}$, used as a device to implicity indicate the growth transformation $x_{n} \rightarrow x$, will now be considered in its continuous form $\bar{g}\left(x, x_{n}\right)$ or $\vec{r}\left(x, x_{n}\right)$. Then using the Heaviside step function $H$ and letting $\Delta x_{n} \rightarrow d x_{n}$, 


$$
\frac{d Q}{d x}=-\frac{4 \pi A}{w} \int_{x_{s}}^{x}\left(\frac{\nu_{c}}{u} I^{*}-N_{g} * \frac{d g^{*}}{d x}\right) \bar{r}^{-2} q_{d}(x, \bar{r}) d x_{n}
$$

and

$$
y_{c}(x, g)=\int_{x_{s}}^{x}\left(\frac{\nu_{c}}{u} I^{*}-N_{g} * \frac{d^{*}}{d x}\right) H(\bar{g}-g) d x_{n},
$$

where $y_{c}$ is the distribution function for the condensate defined congruently to $\mathrm{y}$ and $\mathrm{g}$ may be considered continuous.

Comments

For some types of vapor, the formation of dimers may be important. Thus it may be desirable to expand the model given in this review to account for dimer formation kinetics explicitly. Also, for fixed $T$, as $\mathrm{P} \rightarrow 0$ then $\mathrm{g}^{*} \rightarrow 0$ in the liquid drop model. This implies that a nucleation model for very low pressure should be based on a study of reaction rate kinetics for low order polymers. This kind of approach would be specific to the particular vapor of interest and would lead to considerable complications in the model. Some recent research has been directed at this problem of molecular kinetics. In Ref. 27, a computer molecular dynamics simulation is used to compute dimer formation rates for a gas with atoms interacting with the Lennard-Jones 12-6 potential. Investigation of condensation in a $\mathrm{CO}_{2}$ free jet expansion is reported in Ref. 28. Experimental and theoretical concentrations for $\mathrm{g}=2$ to 5 are shown to have good agreement provided an appropriate accommodation coefficient is chosen.

It should also be noted that there are situations where drop sizes become large and growth under continutum gas conditions is important. An example of this is the experiment in a cloud chamber described in 
Ref. 29 where results are compared to various continuum growth theories. This matter is discussed in detail in Ref. 1.

II. Solutions to the Transient Problem

Conversion of the Nucleation Equation

For high pressure conditions (on the order of an atmosphere) it can be expected that the time required for the nucleation process to relax to the steady state limit is small (Refs, 1,4) and that the transient behavior can be neglected. On the other hand, at low pressures the transient behavior may dominate the nucleation process. (The conditions will be assumed limited to those where $\left.g^{*} \gg 1.\right)$ An example of a low pressure device in which condensation may be important is a metal vapor 1aser (Ref. 30). A numerical treatment of the transient problem is detailed in this part of the report.

The author made a considerable attempt to find an analytical solution to the nucleation equation; this was unsuccessful. Therefore reliance was placed on finding a numerical scheme to integrate the equation. Since the quasi-equilibrium distribution depends exponentially on the droplet free energy, the actual droplet concentration can be expected to depend strongly on the variables $p, T$ and $g$. This is very awkward from the standpoint of numerical integration and a more well behaved function is desired. A natural choice is $\Psi \equiv \ln \mathrm{N}_{\xi}$; the nucleation equation then takes on the nonlinear form

$$
\frac{\partial \Psi}{\partial \theta}=\frac{\partial^{2}}{\partial \xi^{2}}(\Psi+F)+\frac{\partial \Psi}{\partial \xi} \frac{\partial}{\partial \xi}(\Psi+F) \text {. }
$$


(See note below). To solve this equation numerically, it was decided to revert to difference-differential equations analogous to the starting binary collision equations but with a fixed difference interval $\Delta \xi$. The backward and forward differences in variable $g$ are mimicked by biasing the centered difference $\delta$ by an amount $b$ :

$$
\delta_{F}=\frac{1}{2}(1 \mp b) E \pm b-\frac{1}{2}(1 \pm b) E^{-1},
$$

where $E$ and $E^{-1}$ are the raising and lowering shifting (or ladder) operators respectively and $b(\xi)=\sqrt{1.5 / \xi} / \Delta \xi$. Let $\Psi_{0}$ denote the value of $\Psi$ at $\xi_{0}$; $\Psi_{ \pm}$at $\xi_{0} \pm \Delta \xi$. The nucleation equations become for a set of points $\left\{\xi_{0}\right\}$

Note: By performing a Taylor series on $F(\xi)$ at a proper point $\bar{\xi}(\mu)$, it can be shown that a quadratic form well approximates $F$ with absolute error $\leqslant 1$ over a range of drop size that is of physical significance. Correspondingly, the approximate convection velocity in the convectiondiffusion equation for $y$ is linear in $\xi$ and a linear transformation $\xi^{\prime}=A(\theta) \xi+B(\theta), \theta^{\prime}=\theta^{\prime}(\theta)$ yields a simple one dimensional diffusion equation in the new variables. This equation is seemingly tractable. However, the new equation possesses a moving boundary condition whose value and velocity are strong functions of the time variable $\theta^{\prime}$ and the author was unable to generate an analytic solution to the 'simplified' problem. Moreover, the transformation is bad in that for increasing time the range of the variables $\left(\xi^{\prime}, \theta^{\prime}\right)$ corresponding to a given region in $(g, t)$ diminishes rapidly, i.e., it behaves almost as a singular transformation. It was this kind of circumstance that led to the abandonment of analytical efforts and adoption of the nonlinear form. 


$$
\begin{aligned}
& \frac{\mathrm{d} \Psi}{\mathrm{d} \theta}=\Delta \xi^{-2}\left[\Psi_{+}+F_{+}+\Psi_{-}+F_{-}-2\left(\Psi_{0}+F_{0}\right)\right. \\
& \left.+\delta_{-} \Psi_{0} \cdot \delta_{-}\left(\Psi_{0}+F_{0}\right)\right] \text {, } \\
& I_{0}=-\Delta \xi^{-1} \exp \left(\Psi_{0}\right) \delta_{+}\left(\Psi_{0}+F_{0}\right) . \\
& \text { where } \mathrm{E} \Psi_{0} \equiv \Psi_{+} \text {, etc. }
\end{aligned}
$$

To find values at an intermediate point of $\xi$; quadratic interpolation is used. For a given total interval of $\xi$, the number of difference equations (hence $\Delta \xi$ ) can be adjusted until a desired accuracy is obtained. The set of first order differential equations in $\theta$ (or $t$ ) was solved on a UNIVAC 1108 utilizing a JPL Subroutine Library integrator SVDQ (Ref. 31). Comparison with the Numerical Results of Courtney and Clark

Courtney and Clark (Ref. 4) have numerically integrated the binary collision difference-differential equations for water vapor nucleation using sets of 50, 100, and 200 equations. Results from the set of $100(20 \leq \mathrm{g} \leq 119)$ are given in figures and tables in the reference. The pressure and temperature were assumed constant and the accommodation coefficient was taken to be unity. On the upper boundary (e.g., g=120) the concentration $\mathrm{N}_{\mathrm{g}}$ was assumed nu11* and on the lower boundary $(g=19)$ the quasi-equilibrium value was used. The initial concentrations were taken nu11 except for some computations where for $g=20$ the quasiequilibrium value was used. (Some exceptions to these cited boundary and initial conditions were also used with minor effects; for details see Ref. 4) The differences in the initial condition for $g=20$ had * This assumption was shown to have negligible effect. for $g$ values differing by five or more from the highest. 
significant influence on concentrations only for $g>g^{*}$ or very sma11 times $(t \leqslant 0.1 \mu \mathrm{sec})$. As would be expected, this influence was greatest at lower pressure. Computations were carried out on an IBM 7090 computer. The computer time is not disclosed in the reference, but it is stated that "... even on a 7090 was burdensome."

Corresponding calculations were performed using the equations of the previous section. A set of up to 40 equations was used for $10.7 \leq \xi \leq 36.3$ giving $\Delta \xi \geq 0.64$. At the upper boundary it was assumed that $(E-1)\left(\Psi_{0}+F_{0}\right)=-45.0$ (this is nearly equivalent to nulling the upper boundary concentration) and a1so $\delta$ _ was replaced with a backward difference $(b \rightarrow 1)$. Essentially null initial conditions were used: $\left.\{\Psi\}\right|_{t=0}=-88.0$. In testing the computer program it was discovered that a minimum of 20 to 25 equations $(\Delta \xi \leq 1.28)$ was necessary to provide adequate numerical accuracy (error of a few percent in $\mathrm{N}_{\mathrm{g}}$ except for the largest of $g$ ). The accuracy was found to depend quite strongly on the number of equations (or $\Delta \xi$ ). For example, using a set of 16 resulted in as much as order of magnitude error in $\mathrm{N}_{\mathrm{g}}$ for small times. In all calculations, $|\Psi| \geq \theta(10)$. A local absolute integration error control parameter in SVDQ was set at the value $10^{-4}$ (actual error is usually an order of magnitude less, Ref. 31). This was more than adequate, giving four or more place accuracy for $\mathrm{N}_{\mathrm{g}}$ in the time integration - as ascertained by other calculations using $10^{-5}$ error control. Some numerical solutions are compared to those of Courtney and Clark (solid lines) in Figs. 1 through 3. (Except for $\mathrm{g} \leq 50$ in Fig. 3, the Courtney and Clark curves are for a finite initial concentration at $g=20$. The effect of this is slight - deviations in the ordinate $\ll 1$ unit.) Note that vapor 
pressures are given in the captions relative to an atmosphere, $p_{\text {atm }}=1.013 \times 10^{5} \mathrm{~N} / \mathrm{m}^{2}$. Agreement is good for $\mathrm{g}<\mathrm{g}^{*}$. The present calculations give longer relaxation times for large $g$, especially at low pressure, Fig. 3. The transient behavior which is shown in its initial stage in Fig. 3 is more completely detailed in Fig. 4. A calculation with conditions $\left(T=233^{\circ} \mathrm{K}, \mathrm{p} / \mathrm{p}_{\mathrm{S}}=10.0, \omega=12.103\right.$ and $\mathrm{p} / \mathrm{p}_{\text {atm }}=1.84 \times 10^{-3}$ ) intermediate to those related to Figs. $1-4$ gave results similar in appearance to that shown in Fig. 3. The Courtney and clark calculations at these conditions were for a null initial concentration at $\mathrm{g}=20$. Another calculation using 20 equations, $\mathrm{T}=293^{\circ} \mathrm{K}$, $\mathrm{p} / \mathrm{p}_{\mathrm{s}}=5.0, \omega=8.35$ and $\mathrm{p} / \mathrm{p}_{\mathrm{atm}}=0.1156$ shows that steady state $\mathrm{is}$ reached for these particular conditions in about $0.2 \mu \mathrm{sec}$. The computation time for this case with $t \leq 2 \mu \mathrm{sec}$ was 30 seconds. Other computer times were 16 seconds (Fig. $1, t \leq 2 \mu \mathrm{sec}$ ), 14 seconds (Fig. 2, $t \leq 2 \mu \mathrm{sec}$ ) and for the conditions of Figs. 3 and 4,9 seconds to $t=2 \mu \mathrm{sec}$ and 18 seconds to $t=10 \mu \mathrm{sec}$. The computation of the transient zone is fast and apparently much quicker than that of Ref. 4.

The fact that the present method yields longer transient relaxation times for the larger $g$ can probably be attributed to the difficulty with the binary collision equations in handling numerically the corresponding sma11 concentrations. For instance, the use of a relative error control is not desirable with an integrator like SVDQ (Ref. 31); this is probably true for any integrator. Since the value of $\mathrm{N}_{\mathrm{g}}$ for larger $\mathrm{g}$ changes drastically, an absolute error control would lead to inaccuracies and/or long integration times. Use of the logarithmic variable $\Psi(\theta, \xi)$ eliminates this difficulty and also permits fewer equations. 
Relaxation in Terms of Universa1 Parameters

One convenience of the liquid drop model is that nucleation is described in terms of the dimensionless universal variables: time $\theta$, area $\xi$, supersaturation function $\mu(\theta)$ and surface energy function $\omega(\theta)$. $\left(\xi^{*}\right.$ or $g^{*}$ can replace either of the functions.) For constant conditions only two parameters, say $g^{*}$ and $w$, are needed to define the nucleation process for given compatible initial and boundary conditions. In this last section of this report, characteristic times for relaxation from null initial concentrations to (near) steady state are presented. The boundary and initial conditions used are the same as that described in the previous section except the lower boundary is taken at $\xi=4.0(g \approx 4)$. Forty equations were used in the computations with $0.4 \leq \Delta \xi \leq 1.1$. In terms of the maximum flux $I_{\max }^{*}$, a relaxation time $\theta_{R}$ is defined as that (first) time when $I^{*}=0.9 I_{\max }^{*} I_{\max }^{*}$ was found to be approximately the same as the flux, $I_{s s}$, resulting from the steady state limit (Part $I$ ). The parameter $z$ will denote the ratio of the critical to the corresponding quasi-equilibrium concentration at $I^{*}=I_{\max }^{*}$. In the steady state limit, $z=\frac{1}{2}$. The calculations were performed for $\omega=5,10,15,20 ; \mathrm{g}^{*}=10,20$, $30,45,60,80,100$ and for $g^{*}=150 ; \omega=5,10$. Figure 5 gives $\theta_{\mathrm{R}}$ vs. $\mathrm{g}^{*}$ for the various $\omega$. A smooth curve is fitted to the calculated points in this figure. In order to characterize the transient behavior as given by the computer solutions, two nominal relaxation process are defined. The first, to be called "to near steady state," is where $I_{\max }^{*}$ is larger than $I_{s s}$, but only by a few percent, $\left|z-\frac{1}{2}\right| \leqslant 10^{-2}$, and $I^{*}$ remains near $I_{\max }^{*}$ upon its attainment for an interval of time 
$\Delta \theta>\theta(10)$ (no computations were performed for $\theta>200$ ). The second, to be called "non steady state," is where $\mathrm{I}_{\max }^{*}$ differs by more than $10 \%$ from $I_{s s}, z \leqslant 0.4$, and $I^{*}$ drops after attaining $I_{\max }^{*}$ with $I^{*} \geq 0.98 I_{\max }^{*}$ for $\Delta \theta \leqslant 10$. In this second process, the populations for $g \geqslant g^{*}$ pass through the steady state limit values after the maximum flux point is reached and continue to relax toward the quasi-equilibrium distribution. Flux $I^{*}$ can become negative. The second process thus becomes what could be more properly termed a condensation process rather than a mere nucleation process. Of course, the nucleation model equation is too simple to accurately portray any such condensation process where energy considerations are important. Thus there is limited significance to results for the second process for $\theta \gg \theta_{R}$, expecially if $I^{*}<0\left(I^{*}=I_{\max }^{*}\right.$ at a time roughly twice $\left.\theta_{R}\right)$. For $50 \leqslant g^{*} \leqslant 100$, the transient behavior is essentially relaxation to near steady state. However, for $\omega=5$ there was a very slow, or 'adiabatic,' drop in $I^{*}$ after its maximum. For $\mathrm{g}^{*}=150$ and for $\mathrm{g}^{*}=10, \omega=5$ the behavior is non steady state. At $\mathrm{g}^{*}=150, \mathrm{I}_{\max }^{*}$ is slightly greater than $0.6 \mathrm{I}_{\mathrm{ss}}$ for $\omega=5$ and $0.8 \mathrm{I}_{\mathrm{ss}}$ for $\omega=10$. At. $\mathrm{g}^{*}=10, \omega=5, \mathrm{I}_{\max }^{*}$ is slight $1 \mathrm{y}$ greater than $1.2 \mathrm{I}_{\mathrm{ss}}$. The transient behavior at other values of parameters $g^{*}$ and $\omega$ is intermediate to the nominal near and non steady state processes. It features a fairly slow drop in $\mathrm{I}^{*}$ after its maximum with $z=0.45$ to 0.48 . For example, at $g^{*}=10, \omega \geq 10 \mathrm{f} 1 \mathrm{ux} I_{\max }^{*} \simeq 1.1 \mathrm{I}_{\mathrm{ss}}$. In general, the transient behavior in the calculations discussed in this section can be described from a practical point of view as being relaxation to a steady state even though an actual steady state may not be reached. The curves of Fig. 5 provide a means of rough estimation of 
the limit to the validity of steady state nucleation theory, provided only the form $\Delta \phi_{\mathrm{g}} \simeq \mathrm{g}_{\mu} \mathrm{kT}+\mathrm{g}^{2 / 3} \omega \mathrm{kT}$ holds.

\section{References}

1. G. M. Hidy and J. R. Brock, The Dynamics of Aerocolloidal Systems, Pergamon Press, Oxford (1970).

2. J. Frenke1, Kinetic Theory of Liquids, Dover Publications, NY (1955).

3. E. A. Guggenheim, Thermodynamics, North-Holland Publishing Co., Amsterdam (1967).

4. W. G. Courtney and W. J. Clark, Texaco Experiment, Inc., Report TM-1250, (Ju1y 1961).

5. J. Lothe and G. M. Pound, J. Chem. Phys. 45630 (July 1966).

6. F. F. Abraham and G. M. Pound, J. Chem. Phys. 48732 (Jan. 1968).

7. H. Reiss, J. L. Katz and E. R. Cohen, J. Chem. Phys. 485553 (June 1968).

8. Y. Miyazawa, et a1, J. Applied Phys. 42476 (Jan. 1971).

9. R. Kikuchi, J. Statistical Phys. 1 351 (1969).

10. H. Reiss, J. Statistical Phys. $\underline{2} 83$ (1970).

11. A. G. Bashkirov and A. G. Sutugin, J. Statistical Phys. 3 87 (1971).

12. R. Kikuchi, J. Statistical Phys. 3331 (1971).

13. W. G. Courtney, J. Chem. Phys. 352249 (1961).

14. G. D. Stein and P. P. Wegener, J. Chem. Phys. 463685 (1967).

15. D. D. McBride and P. M. Sherman, AIAA J. 101058 (Aug. 1972).

16. P. P. Wegener, J. A. Clumpner and B. J. C. Wu, Phys. Fluids 15 1869 (Nov, 1972).

17. R. H. Heist and H. Reiss, J. Chem. Phys. 59665 (July 1973). 
18. T. Chmielewski and P. M. Sherman, AIAA J. 2789 (Apr. 1970).

19. K. Binder and D. Stauffer, J. Statistical Phys. 649 (1972).

20. A. Bonissent and B. Mutaftschiev, J. Chem. Phys. 583727 (May 1973).

21. J. K. Lee, J. A. Barker and F. F. Abraham, J. Chem. Phys. 583166 (Apr. 1973).

22. D. J. McGinty, J. Chem. Phys. 584733 (June 1973).

23. E. R. Cohen, J. Statistical Phys. 2147 (1970).

24. S. K. Loyalka, J. Chem. Phys. 58354 (Jan. 1973).

25. G. M. Pound, J. Physical and Chemical Ref. Data 1 135 (1972).

26. E. E. Salpeter, J. Chem. Phys. 584331 (May 1973).

27. H. W. Harrison and W. C. Schieve, J. Chem. Phys. 583634 (May 1973).

28. W. G. Dorfeld and J. B. Hudson, J. Chem. Phys. 591253 and 1261 (Aug. 1973).

29. M. A. Vietti and B. G. Schuster, J. Chem. Phys. 591499 (Aug. 1973).

30. G. R. Russe11, N. M. Nerheim and T. J. Pivirotto, App. Phys Lett. 21565 (Dec. 1972).

31. F. T. Krogh, "Variable Order Integrators for the Numerical Solution of Ordinary Differential Equations," JPL Section 314 document (May 1, 1969). 


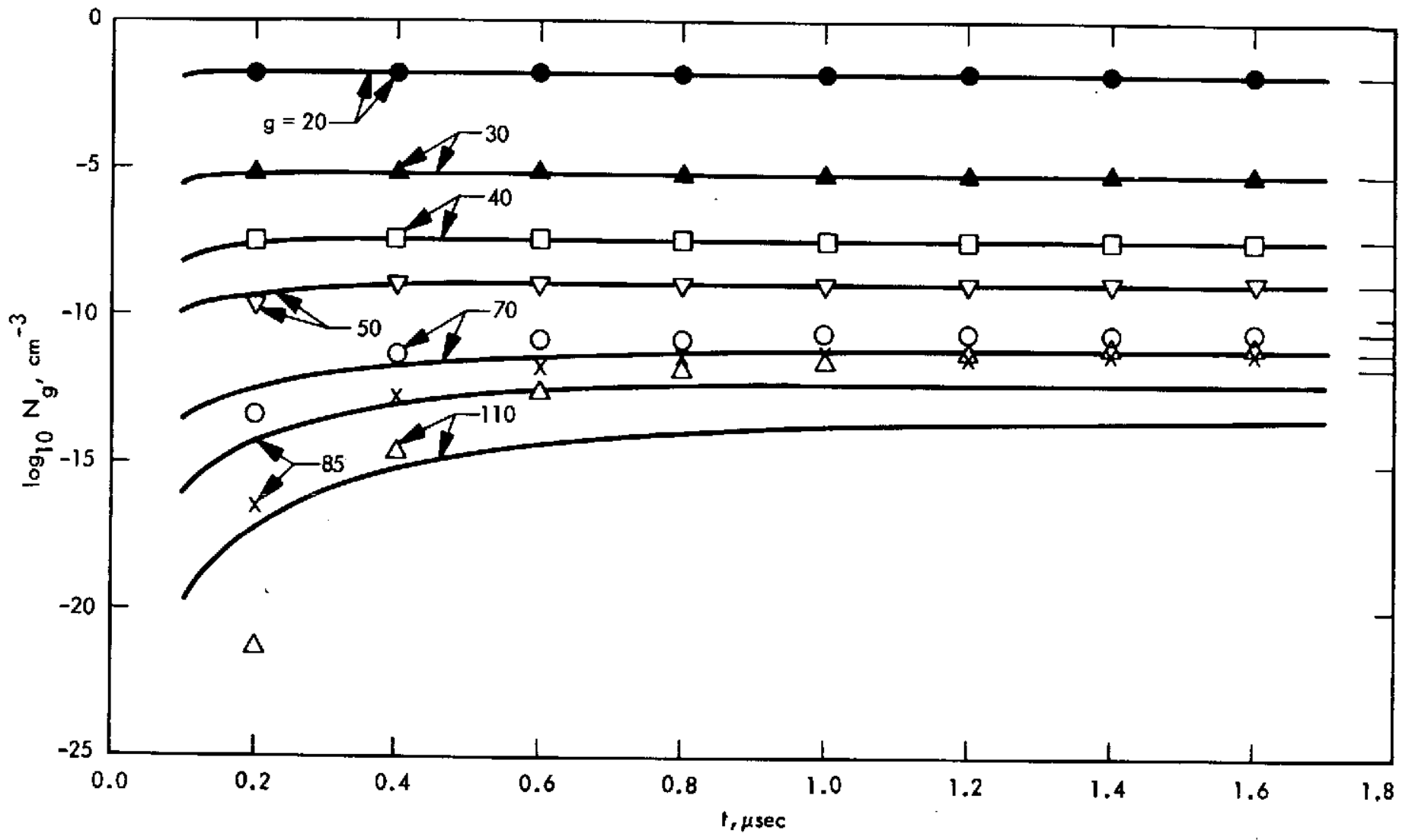

Fig. l. Transient behavior of water droplet concentrations for $\mathrm{p} / \mathrm{p}_{\mathrm{s}}=4.5$ and $\mathrm{T}=263^{\circ} \mathrm{K}$. Solid curves a re from Ref. 4. Tick marks on right border give the steady state limit. $\omega=9.915, \mathrm{~g}^{*}=85,20$ equations, $\mathrm{p} / \mathrm{p}$ atm $=1.27 \times 10^{-2}$ (see text) 


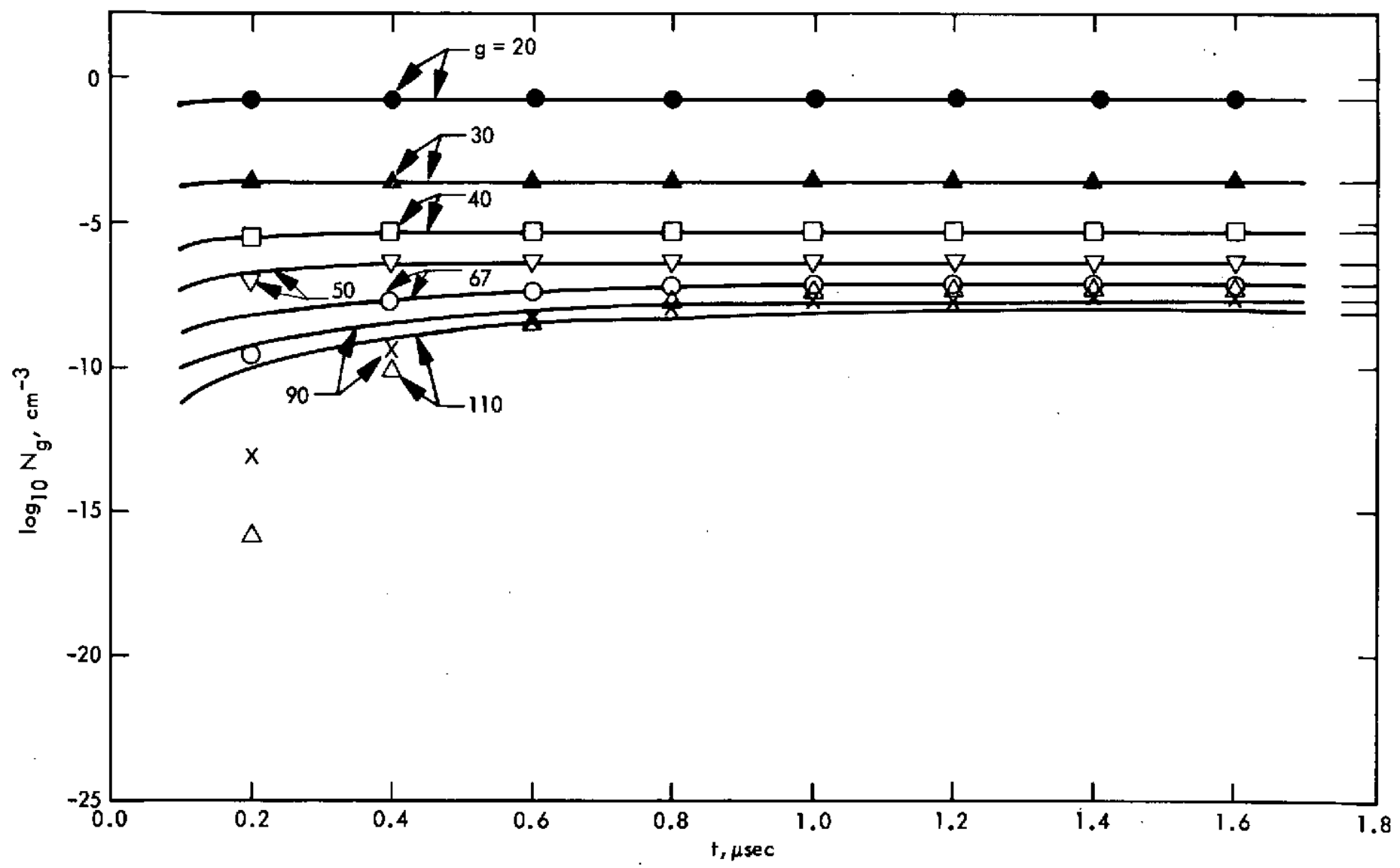

Fig. 2. Transient behavior of water droplet concentrations for $\mathrm{p} / \mathrm{p}_{\mathrm{s}}=5.1$ and $\mathrm{T}=263^{\circ} \mathrm{K}$. Solid curves are from Ref. 4. Tick marks at right give steady state limit. $\omega=9.915, \mathrm{~g}^{*}=67,20$ equations, $\mathrm{p} / \mathrm{p}_{\mathrm{atm}}=1.44 \times 10^{-2}$ 


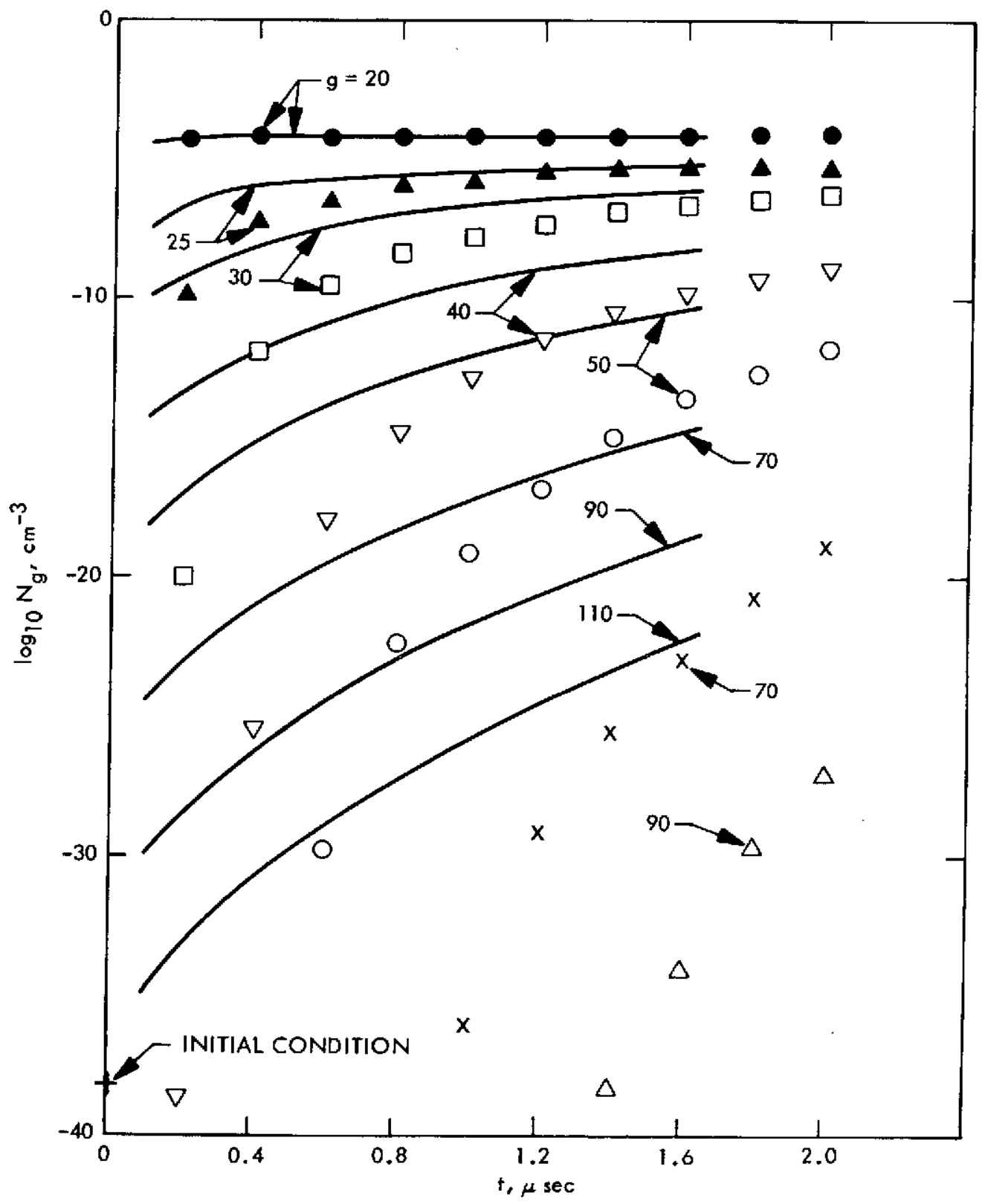

Fig. 3. Transient behavior of water droplet concentrations for $\mathrm{p} / \mathrm{p}_{\mathrm{s}}=20.0$ and $\mathrm{T}=213^{\circ} \mathrm{K}$. Solid curves are from Ref. 4. $\omega=14,045, \mathrm{~g}^{*}=30,25$ equations, $\mathrm{p} / \mathrm{p}_{\mathrm{atm}}=3.95 \times 10^{-4}$ 


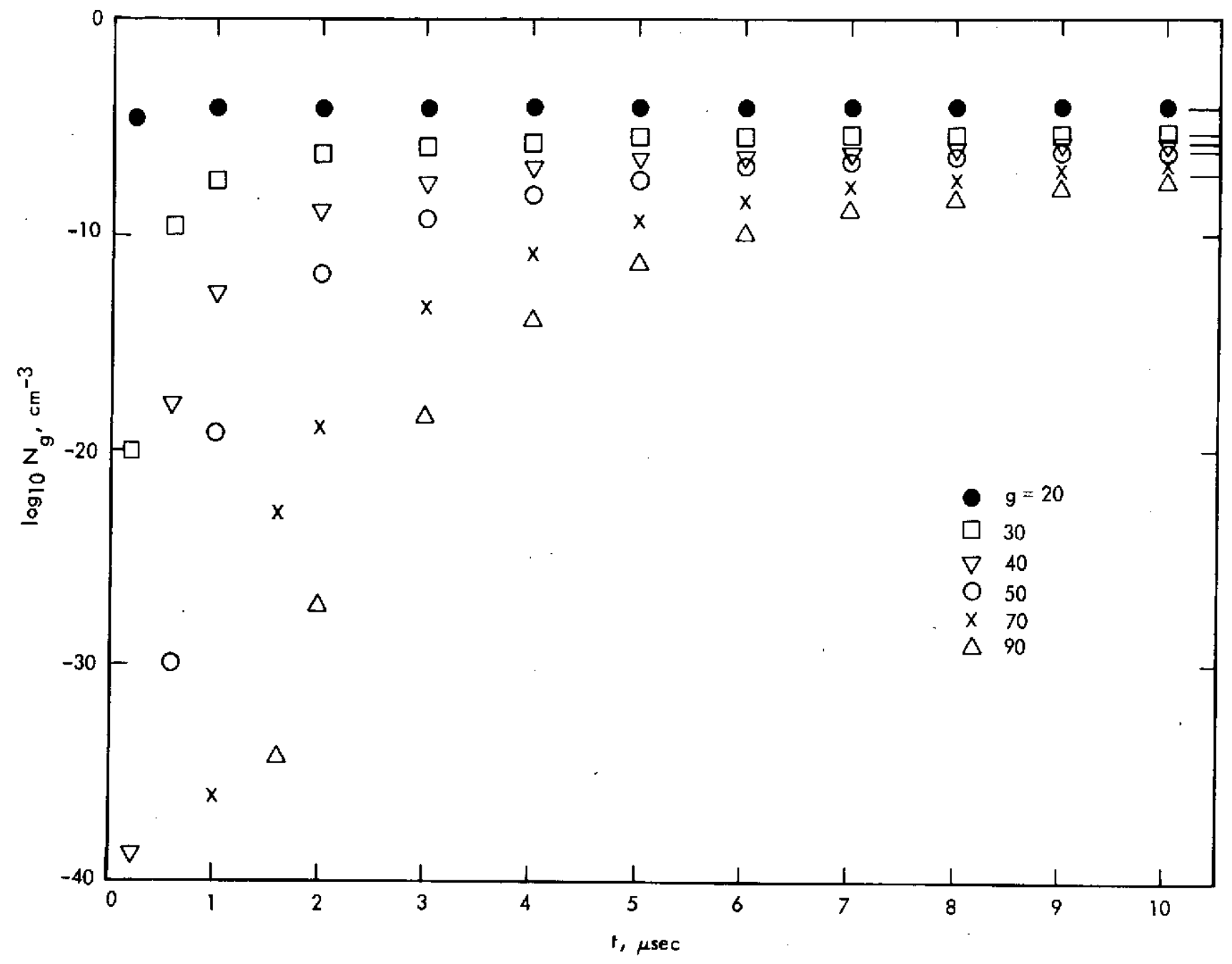

Fig. 4. Transient behavior of water droplet concentrations, same conditions as Fig. 3. Tick marks at right give the steady state limit up to $\mathrm{g}=70$ 


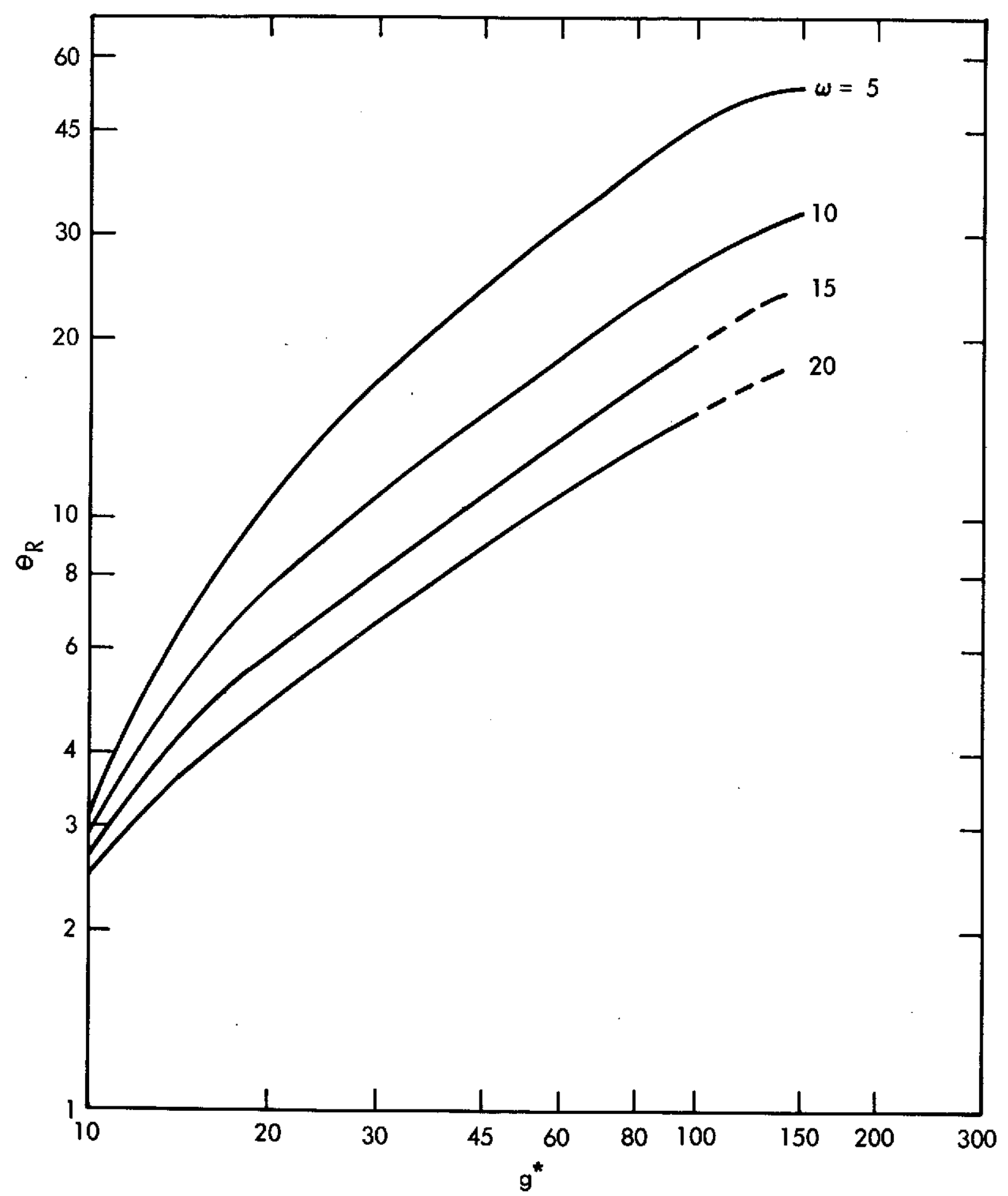

Fig. 5. Nondimensional nucleation relaxation time $\theta_{R}$ vs. critical droplet size $\mathrm{g}^{*}$ for various values of the surface energy parameter $\omega$. (s ee text) 